\title{
Proposal of Walking in Water for ACL Injury Rehabilitation Program by Simulation*
}

\author{
Keiko AKIYAMA**, Motomu NAKASHIMA** and Issei OGASAWARA*** \\ ${ }^{*}$ Graduate School of Information Science and Engineering, Tokyo Institute of Technology, \\ 2-12-1, Ookayama, Meguro-ku, Tokyo, Japan \\ E-mail: motomu@mei.titech.ac.jp \\ ***Japan Institute of Sports Sciences, \\ 3-15-1, Nishigaoka, Kita-ku, Tokyo, Japan
}

\begin{abstract}
The objective of this study was to propose the walking in water for ACL injury rehabilitation program by simulation. For this objective, the simulator which can compute the body load considering the fluid force and force acting on the ACL was utilized. The optimal walking forms for three rehabilitation phases were then computed by the simulation. From the simulation analysis, the form for the first phase of rehabilitation was obtained as "bend the knee all through the swing phase". This form can prevent the ACL from loading the force. The form for the second phase was obtained as "bend the knee greatly and stretch the ankle first, then bend the hip keeping the knee bent and the foot stretched in the swing phase". By this form, the muscles around the knee joint can be trained without loading force on the ACL. The form for the last phase was obtained as "keep the thigh backward and the ankle stretched first, then bend the hip joint quickly and greatly in the swing phase". By this form, the muscles around the knee joint can be trained to the utmost.
\end{abstract}

Key words: Biomechanics, Bio-Motion, Rehabilitation, Sports Biomechanics, Musculoskeletal System, Sports Engineering, Walking in Water

\section{Introduction}

Exercises in water such as swimming and walking are recently becoming popular to various people. In particular, walking in water is easy to perform without any special practices. In the walking in water, the body load due to gravity is cancelled by buoyancy, and some muscles such as hamstrings are recruited greatly due to the fluid force exerted on the lower extremities ${ }^{(1)}$. In addition to this, by changing the walking speed and form, the fluid force can be controlled. Therefore, walking in water is suitable for not only daily exercise but also rehabilitation of sports injuries.

ACL injury ${ }^{(2)(3)(4)}$ is one of the most popular sport injuries. The ACL injury frequently occurs in particular sport scenes, such as landing on one foot, changing the direction quickly, and stopping ${ }^{(5)(6)(7)}$. Surgery or conservation is selected as the treatment for recovery from this injury, although surgery is often recommended for complete recovery. After the immediate post-operative phase, an athletic rehabilitation aimed at returning to sports activity is initiated. Athletic rehabilitation is generally divided into some phases, for example: early rehabilitation phase ( 2 weeks to 4 weeks after the surgery), intermediate phase ( 4 weeks to 3 months after the surgery) and return to activity phase (3 to 6 months after the surgery $)^{(2)(3)(8)(9)(10)(11)}$. In the early rehabilitation phase, the exercise is designed not to stress the ACL graft, since the healing of the ACL graft and the fixation between graft and bone are not sufficient. In the intermediate phase, the exercise which trains muscles 
around the knee without loading large force on the ACL graft is conducted, since it is important to recover the knee muscle strength while keeping the graft safe. In the return to activity phase, exercise aimed at regaining muscle strength to a pre-operative level is carried out to satisfy a sports specific demand. In short, it is important to strengthen the muscles around the knee according to the healing of the ACL graft ${ }^{(12)}$.

In the present rehabilitation for the ACL injury, walking in water is often conducted only during the early rehabilitation phase in order to decrease the body load due to gravity. However, by selecting effective walking forms, not only hamstrings but also knee extensor muscles, such as Vastus medialis and Rectus femoris, can be actively exerted. If such walking forms are suggested, walking in the water will be utilized not only in the beginning but also in all phases of rehabilitation. The selection of walking forms has been conducted subjectively by therapists based on their experience to date. In order to find more effective and safe walking forms, a computer simulation can be an effective tool, since it is possible to systematically obtain the walking form optimized for a given objective function by the simulation. The authors have already developed the widely useable simulator for optimizing the walking form in water ${ }^{(13)}$. With this simulator, it is possible to obtain the optimized walking form by considering the fluid force acting on the body and muscle forces of the lower extremities.

The objective of this study was to propose the walking in water for the ACL injury rehabilitation program by simulation. In this paper, the simulation method is described first. The optimal forms for three rehabilitation phases are then computed by the simulation. Finally, the effects of the optimal forms are discussed.

\section{Simulation Method}

\subsection{Calculation flow}

For the simulation of the present study, the simulator for the optimization of the walking form in water, which has been developed by the authors ${ }^{(13)}$, was utilized. The calculation flow of the simulator is schematically shown in Fig. 1. The simulator consists of three calculation parts: for joint torque, muscle force, and force acting on the ACL. The initial values of the design parameters of the optimization are first set. Next, the joint torques are computed in the calculation part for the joint torque. Then, inputting the joint torque, the muscle forces are computed in the calculation part for the muscle force. The force acting on the ACL is also computed in the calculation part for it, inputting the rectus femoris force and the knee joint angle. The objective function for the optimization is then calculated using the joint torques, muscle forces and the force acting on the ACL. This calculation is repeated until the objective function converges to the maximum value. The Downhill Simplex Method is employed for the optimization. The details of the calculation parts for the joint torque, the muscle force and the force acting on the ACL are explained in the following subsections.

\subsection{The calculation part for the joint torque}

This part has been developed based on the swimming human simulation model SWUM ${ }^{(14)(15)(16)}$. The analytical model is schematically shown in Fig. 2. The human body is defined as a link of the rigid body segments in the simulation model. The motion of the human model (velocity of the center of the mass for the human model and the joint angles) and the ground reaction force (GRF) are input. Then, the inertia force and the gravity exerted on the human model are computed by solving the inverse dynamics problem. In addition to these forces, the fluid force exerted on the human model is computed using the fluid force model. As the sum of these forces, the joint torque and the joint force are calculated. The fluid force was assumed to be computable using the velocity and the acceleration in each part of the body, and it was formulated using the parameters of the fluid force, which were determined by the experiment. The details of the fluid force model are described in the 
previous paper ${ }^{(3)}$. The inputted data, GRF, was measured in an experiment of the previous studies ${ }^{(1)(17)}$. Note that the joint angles only in the sagittal plane were employed in the calculation, since walking is mainly performed in the sagittal plane.

\subsection{The calculation part for the muscle force}

The musculoskeletal model by Yamazaki ${ }^{(18)}$, which is schematically shown in Fig. 3, was utilized for the calculation of the muscle force. In this model, the main eight muscles (GM: gluteus maximus, ILI: iliopsoas, HAM: hamstrings, RF: rectus femoris, VM: vastus medialis, SOL: soleus, GAS: gastrocnemius and TA: tibialis anterior) were modelled as wires. The muscle forces are obtained by solving the optimization problem, in which the sum of squares of the muscle stresses ( $\sum \sigma^{2}$ in Fig. 1) is minimized.

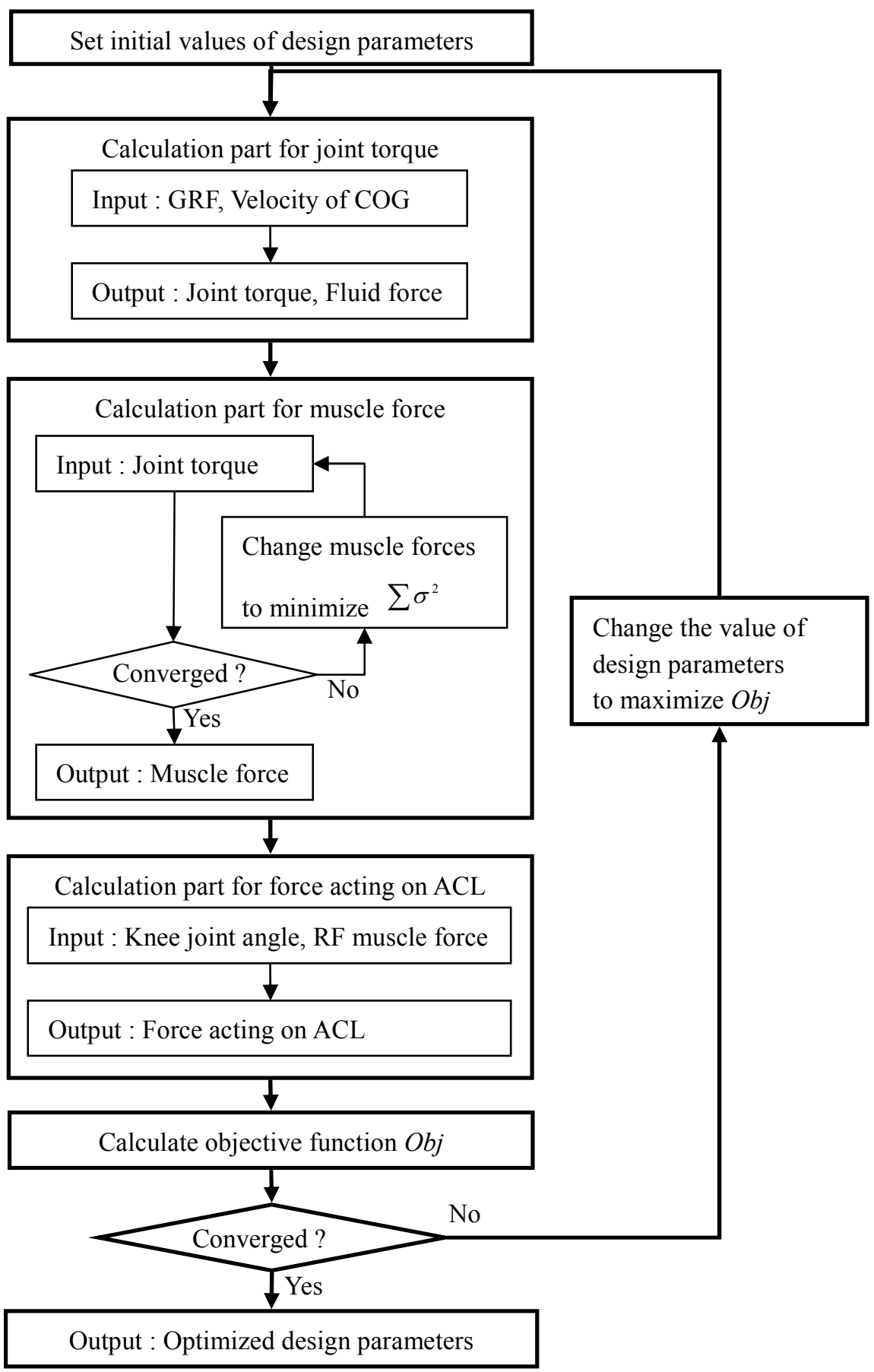

Fig. 1 Calculation flow of the simulator for optimization of walking form in water 


\subsection{The calculation part for the force acting on the ACL}

The biomechanical model of the ACL by Huss et al. ${ }^{(19)}$ was utilized for the calculation of the force acting on the ACL. In this model, the force acting on the ACL is computed by solving the equilibrium formula of forces acting around the knee joint, such as the RF muscle force and the restraining force acting on the tibia. These forces can be expressed as the function of the RF muscle force and knee joint angle. Therefore, the force acting on the ACL can be represented as the function of the RF muscle force and the knee joint angle. In order to obtain the force acting on the ACL simply, the force acting on the ACL was approximated by a polynomial. The approximated force is shown in Fig. 4. It can be seen that the force increases according to the increase of the muscle force of the RF when the

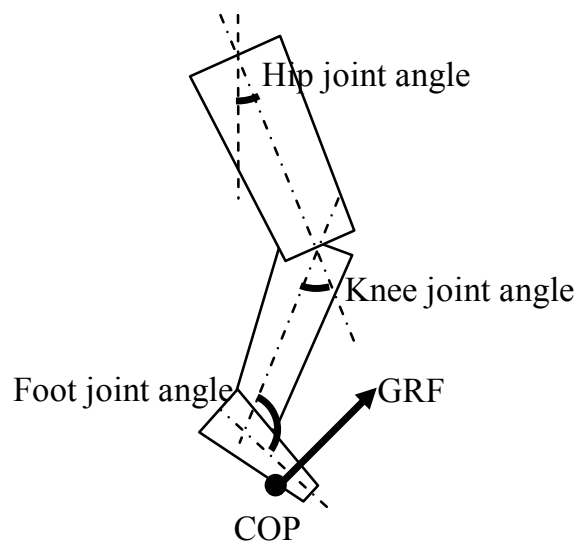

Fig. 2 Analytical model of calculation part for joint torque

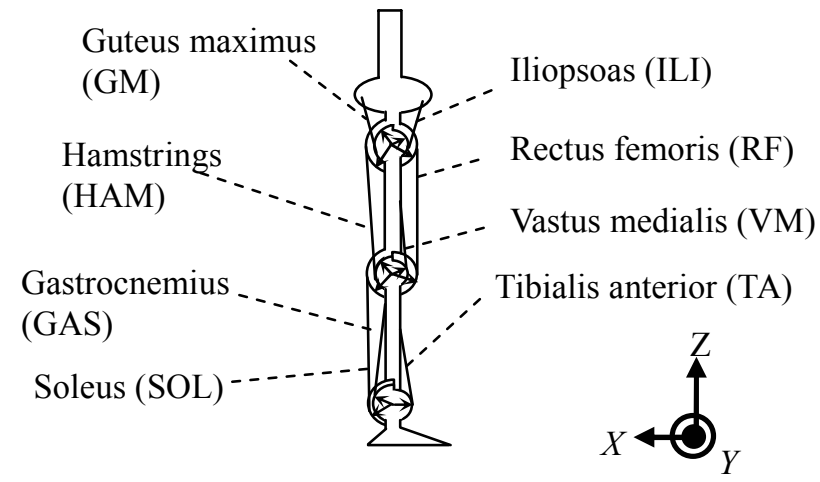

Fig. 3 Musculoskeletal model of calculation part for muscle force

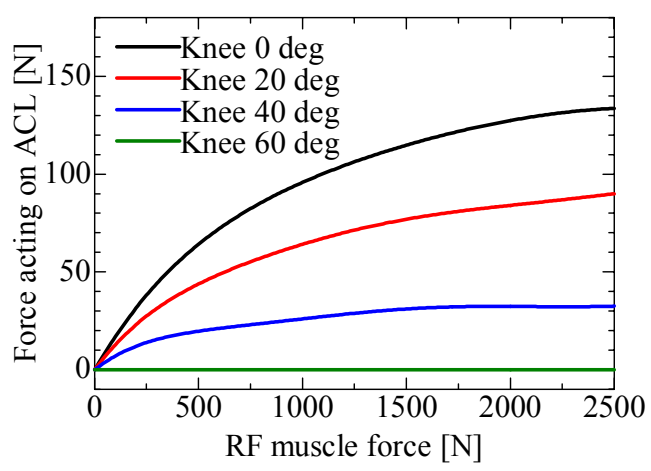

Fig. 4 The relationship between the RF muscle force and force on the ACL 
knee joint angle is below 60 degrees. It is noted that the force becomes zero when the knee joint is over 60 degrees. This results are agreed with the data of Beynnon et al ${ }^{(20)}$, Yagi et al ${ }^{(21)}$ and Markolf ${ }^{(22)}$. They measured the strain of the ACL in vivo or in vitro. From these studies, the ACL can be prevented from loading by bending the knee over 60 degrees.

\section{Conditions of Optimization}

\subsection{Design parameters}

The joint angles (hip, knee and ankle) are set as the design parameters of the optimization. The scheme of the design parameters are shown in Fig. 5. Each joint angle in one cycle is represented by ten control points at constant intervals and a curve interpolating the control points. The former six control points correspond to the stance phase, and the latter four correspond to the swing phase. The joint angles of four points in the swing phase are set as design parameters in the optimization. Therefore, the number of design parameters is twelve in total (four points for three joints).

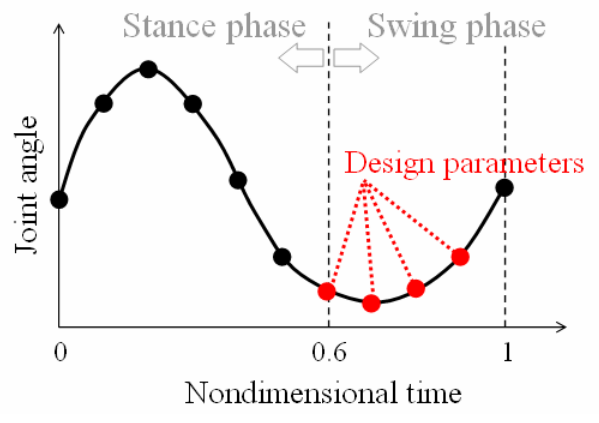

Fig. 5 Scheme of the design parameters

\subsection{Objective function}

\subsubsection{Early rehabilitation phase}

In the early rehabilitation phase, it is important to reduce the force acting on the ACL. Therefore, the optimal form in this phase was defined as the form in which the force on the ACL is minimized. Based on this definition, the objective function was formulated as below:

$$
O b j=\frac{\int_{t=0}^{1} A C L_{\text {Normal }}}{\int_{t=0}^{1} A C L_{\text {New }}}
$$

( $A C L$ : Force acting on the ACL, Normal : Normal form, ${ }_{\text {New }}:$ New form )

The symbol $t$ is nondimensional time normalized by cycle, and $\int_{t=0}^{1}$ represents the integral with respect to time for one cycle. Since this function is the ratio of the force acting on the ACL of the normal and new forms, this function will be maximized by minimizing the denominator. That is, the force acting on the ACL is minimized by maximizing this objective function.

\subsubsection{Intermediate phase}

In the intermediate phase, it is important to strengthen muscles around knee joint, such as the HAM, the VM and the RF. Since the VM and the RF are less exerted compared to the HAM during walking in water, as discussed in the Introduction, it is important to train the VM and the RF. On the other hand, it is also important not to load force on the ACL. Therefore, the optimal form in this phase was defined as the form in which the VM and the RF can be trained without loading force on the ACL. Based on this definition, the objective function was formulated as below: 
$O b j=\frac{\int_{t=0}^{1} F_{V M}}{P C S A_{V M}}+\frac{\int_{t=0}^{1} F_{R F}}{P C S A_{R F}}-W_{A C L} \int_{t=0}^{1} A C L$

$C_{V M}:$ Muscle force of the VM

$F_{R F} \quad$ : Muscle force of the RF

$P C S A$ : Physiological cross sectional area of the muscle

$W_{A C L}$ : Weighting coefficient of force acting on the ACL

In the objective function, the first term is the integral for muscle stress of the VM in one cycle, the second term is the integral for the muscle stress of the RF in one cycle, and the third term is the integral for the force acting on the ACL multiplied by the weighting coefficient.

\subsubsection{Return to activity phase}

In the return to activity phase, it is most important to strengthen muscles for the return. The force acting on the ACL is not so important. Therefore, the optimal form in this phase was defined as the form in which the VM and the RF are greatly trained, not considering the force on the ACL. Based on this definition, the objective function was formulated as below:

$$
O b j=\frac{\int_{t=0}^{1} F_{V M}}{P C S A_{V M}}+\frac{\int_{t=0}^{1} F_{R F}}{P C S A_{R F}}
$$

The first and second terms in Eq. (3) are the same as those in Eq. (2).

\subsection{Constraint condition}

The penalty function was utilized so that the joint angles and joint torques do not exceed their determined maximum values. If the joint angle or torque exceeds their maximum values, the excess amount is subtracted from the objective function. The maximum values of the joint angles were determined, based on the joint range of motion in the human characteristics database ${ }^{(23)}$. The maximum values of the joint torques were shown in Table 1. In Table 1, the ratios to the maximum voluntary contraction ${ }^{(24)}$ are shown in the parentheses. These ratios are the target values for recovery in each phase ${ }^{(2)}$.

Table 1 Maximum joint torques

\begin{tabular}{llcc}
\hline & & Flexion $[\mathrm{Nm}]$ & Extension $[\mathrm{Nm}]$ \\
\hline Hip joint torque $[\mathrm{Nm}]$ & All phases & 99.9 & 74.3 \\
\hline Knee joint torque $[\mathrm{Nm}]$ & Early rehabilitation phase & $28.4(50 \%)$ & $19.1(50 \%)$ \\
\cline { 2 - 4 } & Intermediate phase & $45.5(80 \%)$ & $26.8(70 \%)$ \\
\cline { 2 - 4 } & Return to activity phase & $56.8(100 \%)$ & $34.4(90 \%)$ \\
\hline Ankle joint torque $[\mathrm{Nm}]$ & All phases & 47.7 & 25.6 \\
\hline
\end{tabular}

\subsection{Other conditions}

The body geometry of the model was based on the size of a 20 year old Japanese male obtained from the AIST database of human size and shape ${ }^{(24)}$. The initial values of design parameters were set to the values of the joint angles in the normal walking form. The joint angles in the normal form were taken from the previous studies ${ }^{(1)(17)}$.

\section{Results and Discussion}

Iterations of 400-700 times were required in order to obtain the solutions of the optimization. The calculation time was $10-15$ hours by a $1.2 \mathrm{GHz}$ PC. The forms before and after the optimization for the three phases are shown in Fig. 6 . The red lines emitting from 


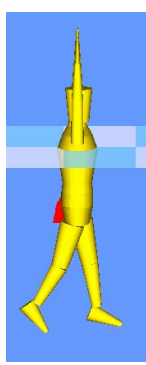

(i) $t=0.6$

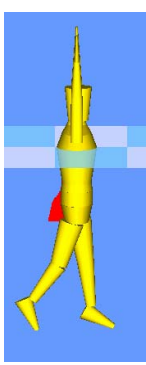

(ii) $t=0.65$

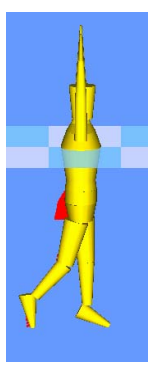

(iii) $t=0.7$

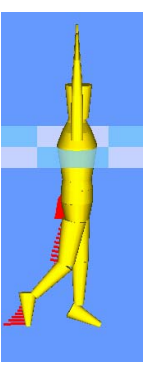

(iv) $t=0.75$

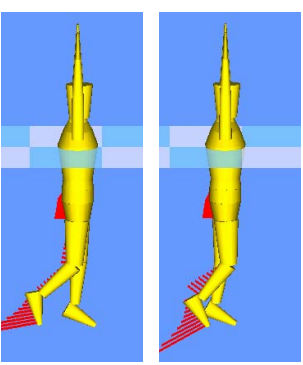

(v) $t=0.8(\mathrm{vi}) t=0.85$
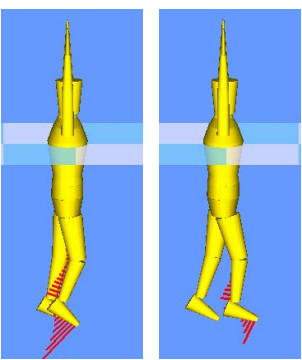

(a) Before optimization

(vii) $t=0.9$ (viii) $t=0.95$

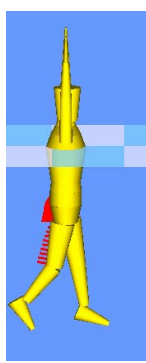

(i) $t=0.6$

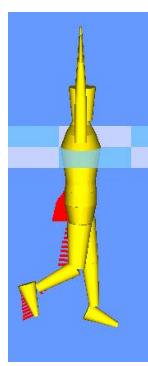

(ii) $t=0.65$

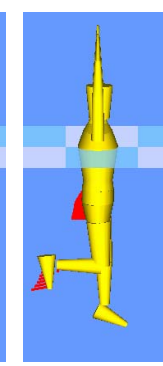

(iii) $t=0.7$

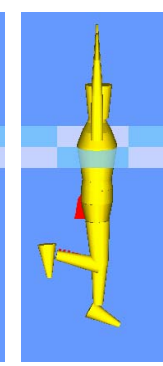

(iv) $t=0.75$

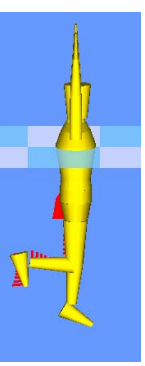

(v) $t=0.8$ (vi) $t=0.85$

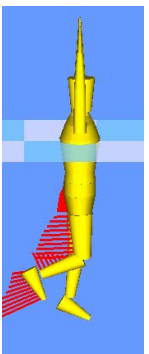

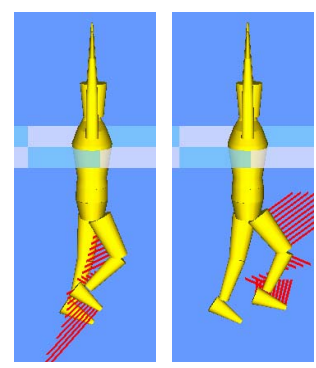

(vii) $t=0.9$ (viii) $t=0.95$

(b) Optimized form for early rehabilitation phase

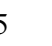


each part of the body represent the points of applications, directions and magnitudes of the fluid forces acting on the body. The joint angles, the muscle forces and the force acting on the ACL for the right leg before and after optimization in one cycle are shown in Fig. 7, 8 and 9 , respectively. The details of each form are discussed in each following subsection.

\subsection{Before optimization (original normal walking form)}

As shown in Fig. 8 (black line), the ACL is loaded in the swing phase $(t=0.6-1.0)$ in this case. This is because the knee joint angle is below 60 degrees (Fig. 7(b)) and the RF is exerted (Fig. 9 (b)). The hip joint is below 20 degrees in this case.

\subsection{Early rehabilitation phase}

Since the objective of the optimization for this phase is to minimize the force acting on the ACL, the force on the ACL becomes zero almost all through the cycle, as shown in Fig. 8 (red line). This is because the knee joint is over 60 degrees at $t=0.65-0.95$ (Fig. 7(b)). Therefore, the optimal form for the early rehabilitation phase is concluded to be "bend the knee all through the swing phase".

\subsection{Intermediate phase}

Since the objective for this phase is to exert the VM and the RF muscle forces without loading large force on the ACL, the VM and the RF muscle forces become much larger than during the phase of protection and before optimization as shown in Fig. 9 (blue lines). The force acting on the ACL becomes larger than the early rehabilitation phase at $t=0.6$, but much smaller than before optimization at $t=0.65-0.95$ (Fig. 8). The VM muscle force is exerted at $t=0.8-1.0$ (Fig. 9(a)), since the ankle joint is extended to be perpendicular to the moving direction (Fig. 6(c)). By this motion, large fluid force acts on the foot, and the VM exerted against this fluid force. The RF is also exerted at the same moment, since the fluid force acts on the foot and thigh. The fluid force acting on the thigh is caused by the motion of hip flexion (Fig. 6 (c)). On the other hand, the force acting on the ACL is very small. This is because the knee joint is over 60 degrees at $t=0.7-0.95$ (Fig. 7 (b)). Therefore, the optimal form for the intermediate phase is concluded to be "bend the knee greatly and stretch the ankle first, then bend the hip keeping the knee bent and the foot stretched in the swing phase".

\subsection{Return to activity phase}

Since the objective for this phase is to exert the VM and the RF muscle forces to the utmost, the VM and the RF muscle forces become the greatest among all phases (green lines, Fig. 9). Instead, the force acting on the ACL becomes the greatest among all phases (Fig. 8). The VM muscle force is exerted at $t=0.7-0.9$ (Fig. 9(a)), since the ankle joint is extended to be perpendicular to the moving direction (Fig. 6(d)) and the knee joint is more extended than that in the intermediate phase. In addition to this, the hip joint once extends around $t=0.7$ (the thigh moves backward), and flexes from 20 to 80 degrees, which is a much larger range than that in the intermediate phase. By these motions, the large fluid force acts on the shank and foot, and the VM exerted against this fluid force. The RF is also exerted at the same moment, since the large fluid force acts on the whole of lower extremities. Instead, force acting on the ACL is the greatest, especially at $t=0.7-0.9$ (Fig. 8). This is because the knee joint is below 60 degrees at this moment. Therefore, the optimal form for the return to activity phase is concluded to be "move the thigh backward and stretch the ankle first, then bend the hip joint quickly and greatly in the swing phase". 


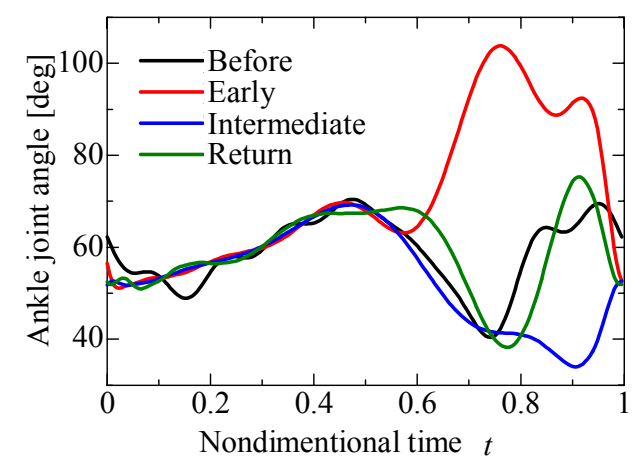

(a) Ankle joint

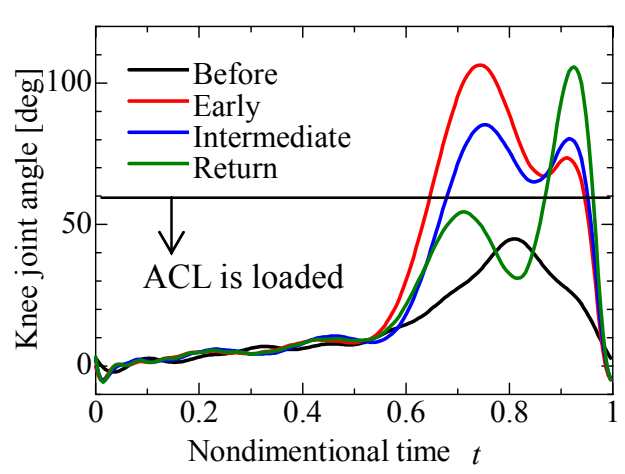

(b) Knee joint

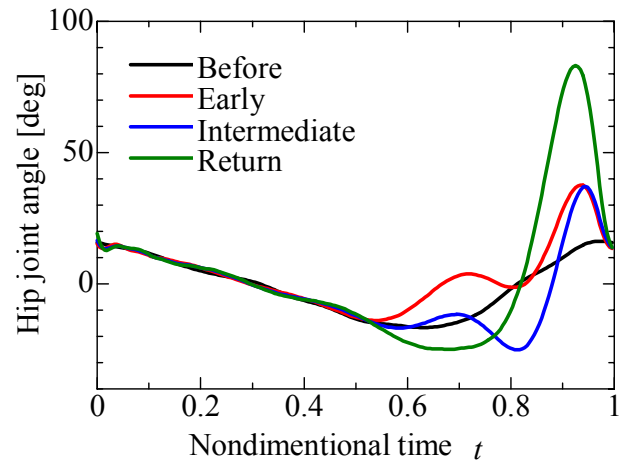

(c) Hip joint

Fig. 7 Time history of joint angle

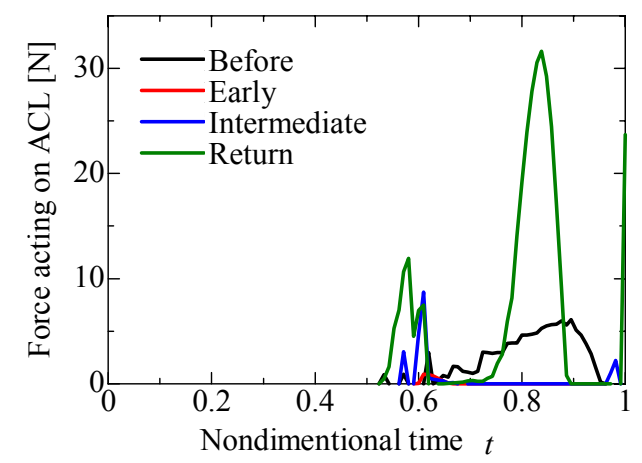

Fig. 8 Time history of force acting on the ACL

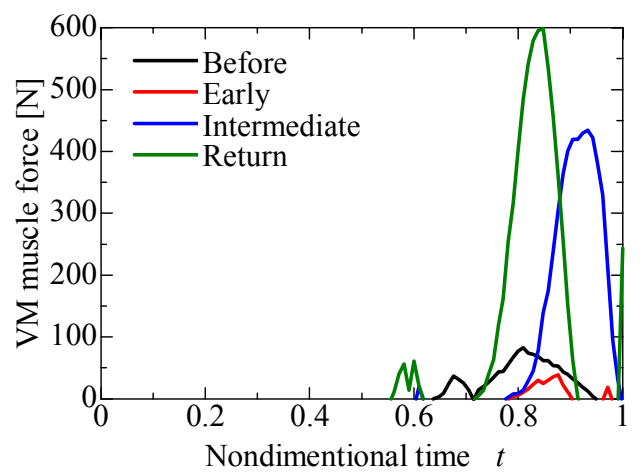

(a) VM

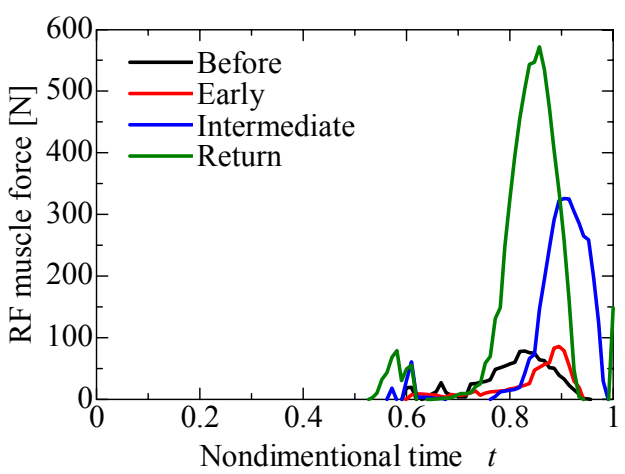

(b) RF

Fig. 9 Time history of muscle force 


\section{Conclusions}

The walking program in water for the rehabilitation of the ACL injuries was obtained by the simulations in the present study. The results are summarized as follows:

(1) In the early rehabilitation phase, the obtained form is concluded to be "bend the knee all through the swing phase". By this motion, the force acting on the ACL can be zero almost all through the cycle.

(2) In the intermediate phase, the obtained form is concluded to be "bend the knee greatly and stretch the ankle first, then bend the hip keeping the knee bent and the foot stretched in the swing phase". By this motion, the VM and the RF muscle can be strengthened without loading large force on the ACL.

(3) In the return to activity phase, the obtained form is concluded to be "move the thigh backward and stretch the ankle first, then bend the hip joint quickly and greatly in the swing phase". By this motion, the VM and the RF muscle can be fully strengthened.

As the future task, it will be necessary to validate the effectiveness of these walking forms by an experiment using subjects.

\section{References}

(1) Akiyama, K., Nakashima, M. and Miyoshi, T., Simulation Analysis of Mechanical Body Load during Walking in Water, Transactions of the Japan Society of Mechanical Engineers, Series C, Vol. 76, No. 763 (2010), pp. 673-681.

(2) Fukubayashi, T., Guide for the athletic rehabilitation, Bunkodo (2008), pp.114-127.

(3) Bousquet, G., Beguec, L. P. and Girardin, P. (Original authors), Shiota, E., Yuge, I. and Ihara, K. (Translators), Functional anatomy and ligament injury of knee (in Japanese), Kyodoisho (1995), pp.230-234.

(4) Renstrom, P., Ljungqvist, A., Arendt, E., Beynnon, B., Fukubayashi, T., Garrett, W., Georgoulis, T., Hewett, E. T., Johnson, R., Krosshaug, T., Mandelbaum, B., Micheli, L., Myklebust, G., Roos, E., Roos, H., Schamasch, P., Shultz, S., Wemer, S., Wojtys, E. and Engebretsen, L., Non-contact ACL Injuries in Female Athletes : An International Olympic Committee Current Concepts Statement, British Journal of Sports Medicine, Vol. 42, (2008), pp. 394-412.

(5) Olsen, O., Myklebyst, G., Engebrestsen, L. and Bahr, R., Injury Mechanisms for Anterior Cruciate Ligament Injuries in Team Handball, A systematic Video Analysis, The American Journal of Sports Medicine, Vol. 32, No. 4, (2004), pp. 1002-1012.

(6) Hewett, E. T., Myer, D. G. and Ford, R. K., Anterior Cruciate Ligament Injuries in Female Athletes, Part 1, Mechanisms and Risk Factors, The American Journal of Sports Medicine, Vol. 34, No. 2, (2006), pp. 299-311.

(7) Krosshaug, T., Nakamae, A., Boden, P. B., Engebretsen, L. Smith, G., Slauterbeck, R. J., Hewett, E. T. and Bahr, R., Mechanisms of Anterior Cruciate Ligament Injury in Basketball, Video Analysis of 39 Cases, The American Journal of Sports Medicine, Vol. 35, No. 3, (2007), pp. 359-367.

(8) Wilk, E. K., Arrigo, C., Andrews, R. J. and Clancy, G. W., Rehabilitation After Anterior Cruciate Ligament Reconstruction in the Female Athlete, Journal of Athletic Training, Vol. 34, No. 2, (1999), pp. 177-193.

(9) Paulos, L., Wnorowski, D. and Beck, C., Rehabilitation Following Knee Surgery, Recommendations, Sports Medicine, Vol. 11, No. 4, (1991), pp. 257-275.

(10) Smekal, D.,Kalina, R. and Urban, J., Rehabilitation After Arthroscopic Anterior Cruciate Ligament Reconstruction, Acta Chirurgiae orthopaedicae et traumatologiae Cechoslovaca, Vol. 73, No. 6, (2006), pp. 421-428.

(11) Tovin, J. B., Wolf, L.S, Greenfield, H. B., Crouse, J. and Woodfin, A. B., Comparison of the Effects of Exercise in Water and on Land on the Rehabilitation of Patients With Intra-articular Anterior Cruciate Ligament Reconstructions, Physical Therapy, Vol. 74, No. 
8, (1994), pp. 710-719.

(12) Paulos, L., Noyes, R. F., Grood, E. and Butler, L. D., Knee Rehabilitation After Anterior Cruciate Ligament Reconstruction and Repair, The American Journal of Sports Medicine, Vol. 9, (1981), pp. 140-149.

(13) Akiyama, K. and Nakashima M., Development of Widely Useable Simulator for Optimization of Walking Form in Water, Proceedings of the 8th Conference of the International Sports Engineering Association, (2010), (to be published).

(14) Nakashima, M., Sato, K. and Miura, Y., Development of Swimming Human Simulation Model Considering Rigid Body Dynamics and Unsteady Fluid Force for Whole Body, Journal of Fluid Science and Technology, Vol. 2, No. 1 (2007), pp. 56-67.

(15) Nakashima, M., Mechanical Study of Standard Six Beat Front Crawl Swimming by Using Swimming Human Simulation Model, Journal of Fluid Science and Technology, Vol. 2, No.1 (2007), pp.290-301.

(16) SWUM Developer Team, "Swimming Human Simulation Model SWUM". SWUM Website. (online). available from <http://www.swum.org/>, (accessed 2009-10-21).

(17) Miyoshi, T., Shirota, T., Yamamoto, S., Nakazawa, K. and Akai, M., Lower Limb Joint Moment during Walking in Water, Disability and Rehabilitation, Vol. 25, No.21 (2003), pp. 1219-1223.

(18) Yamazaki, N., Analytical Model and Simulation of walking, Biomechanism (in Japanese), Vol. 3 (1975), pp. 261-269.

(19) Huss, R. A., Holstein, H. and J. O' Connor J., A mathematical model of forces in the knee under isometric quadriceps contractions, Clinical Biomechanics, Vol. 15 (2000), pp.112-122.

(20) Beynnon, D. B. and Fleming, C. B., Anterior cruciate ligament strain in-vivo: A review of previous work, Journal of Biomechanics, Vol. 31 (1998), pp. 519-525.

(21) Yagi, M., Wong, D. E., Kanamori, A., Debski, E. R., Fu, H. F. and Woo, L-Y. S., Biomechanical Analysis of an Anatomic Anterior Cruciate Ligament Reconstruction, The American Journal of Sports Medicine, Vol. 30, No. 5 (2002), pp. 660-666.

(22) Markolf, L. K., Gorek, F. J., Kabo, M. and Shapiro, S. M., Direct Measurement of Resultant Forces in the Anterior Cruciate Ligament, The Journal of Bone and Joint Surgery, Vol. 72 (1990), pp. 557-567.

(23) National Institute of Technology and Evaluation, "Human characteristics database". National Institute of Technology and Evaluation Website. (Online). available from < http://www.tech.nite.go.jp/human/index.html >, (accessed 2010-01-20).

(24) AIST Digital Human Research Center, "AIST database of human size and shape". AIST Website. (Online). available from <http://riodb.idase.aist.go.jp/dhbodydb/index.php.ja>, (accessed 2010-01-20). 